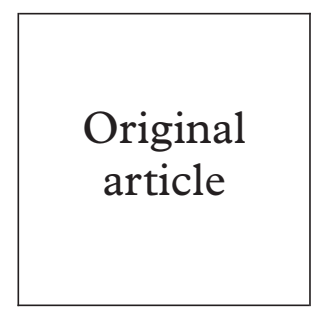

\section{The Bali STD/AIDS study: association between vaginal hygiene practices and STDs among sex workers}

\author{
Barbara D Reed, Kathleen Ford, Dewa N Wirawan
}

Objectives: To assess the association between genital cleansing practices and the prevalence of sexually transmitted diseases and of sexual health knowledge among female sex workers in Bali, Indonesia.

Methods: Low priced commercial sex workers (CSWs) participated in the Bali STD/AIDS Study, a 3 year educational project evaluating the effect of education on the subsequent use of condoms and the prevalence of STDs and AIDS. Structured interviews, genital evaluation, laboratory evaluation for STDs, and treatment were performed. Vaginal cleansing characteristics including frequency, type, and reasons for use, were evaluated. Associations between these characteristics and the presence of bacterial and viral genital infections were assessed.

Results: Of 625 female sex workers evaluated between May and July 1998, 99.1\% used substances, such as soap and toothpaste, to clean the vagina at least daily, with $69.3 \%$ performing this after each intercourse. The women using such cleansers after each client did not differ from those using them once or less daily in education, AIDS and condom use knowledge, time working as a CSW, or number of clients in the previous day. However, they were younger than those using vaginal cleansers daily or less, and reported lower condom usage in the past week. Several genital symptoms, such as discoloured discharge and odour, were reported less by women with the highest frequency of vaginal cleanser use. Prevalence of genital infections in this population of women was substantial, with bacterial infections more prevalent than viral infections. Infections were not associated with the type of cleanser used, using a genital cleanser on the day of examination, or using a cleanser after each client versus daily or less, except for candida colonisation, which was more prevalent in women cleansing after each client $(\mathrm{OR}=1.87,95 \% \mathrm{CI}$ $1.21,2.90$ ). However, symptomatic candida vulvovaginitis (positive culture plus presence of symptoms) was not associated with the prevalence of genital cleansing. Women using genital cleansing in part for "infection avoidance" (39.7\%) were more likely to have heard of STDs and AIDS, but were less knowledgeable about these infections, compared with women not citing this reason for genital cleansing.

Conclusion: Commercial sex workers in low priced brothels in Bali have a high rate of genital infections, with lower rates of viral compared with bacterial infections. Genital cleansers, on a daily or after each intercourse schedule, are used routinely. Although genital cleansing after each intercourse was associated with fewer genital symptoms, the prevalence of STDs did not differ significantly based on this frequency, and the women's knowledge of STDs and AIDS was less than that of women cleansing less often. The effect of genital cleansing in general on STD and AIDS prevalence could not be assessed in this population owing to the lack of a non-cleansing cohort. Further study to elucidate the effect of vaginal cleansing practices on STD prevalence and resistance is needed.

University of Michigan Department of Family Medicine, University of Michigan School of Public Health, Ann Arbor, MI, USA

B D Reed

\section{Department of Epidemiology, Denpasar, Bali, Indonesia \\ K Ford}

Kerti Praja

Foundation, Denpasar, Bali, Indonesia

D N Wirawan

Correspondence to: Dr Reed, University of Michigan, 1018 Fuller Street, Ann Arbor, MI, 48109, USA

barbr@umich.edu

(Sex Transm Inf 2001;77:46-52)

Keywords: genital cleansing; sexually transmitted diseases; douching; Bali

\section{Introduction}

Genital cleansing is a common practice in many populations around the world..$^{1-4}$ Despite this, the effect of this practice on susceptibility and resistance to genital infections and AIDS is not well elucidated, although increased risks of STDs, ${ }^{15-7}$ pelvic inflammatory disease (PID),${ }^{8-10}$ and of ectopic pregnancies ${ }^{811}$ have been previously reported. Furthermore, the characteristics of women using genital cleansing on a routine basis are not yet clearly described. Interest in vaginal cleansers has increased as studies indicated microbicides used in the vagina, such as nonoxynol-9, had virucidal activity against non-encapsulated viruses, including $\mathrm{HSV}-2$ and HIV-1, and against Chlamydia trachomatis, but no activity against encapsulated viruses, such as human papillomavirus. ${ }^{12-14}$ A recent report suggests sodium dodecyl sulphate (SDS), a surfactant that also has protein denaturing capabilities, may have virucidal activity against human papillomavirus (HPV) as well as HIV-1 and HSV$2 .^{12}$ This compound is present in significant concentrations in many toothpastes, soaps, and shampoos, substances often used as vaginal cleansers throughout the world.

The Bali AIDS/STDs Study was conducted between 1997 and 2000. In our evaluation of commercial sex workers in Bali, Indonesia, we found them to be routinely cleansing the vaginal and vulvar area after sex with clients 
or partners, using agents such as soaps and toothpastes. We, therefore, evaluated characteristics of this cleansing practice detailed at the 6 month follow up round of data collection, and assessed the association between these cleansing practices and genital symptoms, rates of bacterial and viral infections, and knowledge of STDs and AIDS among these women.

\section{Methods}

The Bali STD/AIDS Study is an ongoing field study of female commercial sex workers (CSWs) working in low priced brothels in the Denpasar area of Bali, Indonesia. The project is designed to evaluate the effect of an educational intervention of two intensity levels on the extent of knowledge regarding sexually transmitted diseases (STDs), AIDS, and condom use, and, in turn, the effect on the prevalences of reported condom use and of detected STDs and other genital infections. The project was approved by the institutional review boards of the School of Public Health at the University of Michigan, Ann Arbor, USA, and of the Kerti Praja Foundation, Denpasar, Bali.

STUDY POPULATION

Participants included all women working in five geographical groupings of low priced commercial sex workers within a 10 mile radius of central Denpasar, Bali. Women who were members of the brothels, but were unavailable on the day of testing, were contacted within a 2 week period and enrolled at that time. No exclusions were made. All women were interviewed but 28 women in the initial evaluation and 18 women at the first follow up evaluation did not appear for the physical examination. Informed consent was obtained orally by the field workers at the brothel sites where the interview was conducted, and again by the physicians at the clinic before the examination and specimen collection.

\section{HISTORY AND PHYSICAL EXAMINATION}

Extensive evaluation, including historical information, physical examination data, and inhouse and referral laboratory data, was conducted every 6 months for a total of four rounds. Trained field workers from the Kerti Praja Foundation in Denpasar, Bali, Indonesia, visited the workers in their place of work and obtained informed consent from the women and from their pimps. The field workers then conducted scripted interviews with each woman regarding epidemiological data, knowledge level regarding STDs and AIDS, sexual behaviours, past genital infections and treatments, and acute genital symptoms. The women were then transported to the clinical testing site for an educational session regarding STDs, AIDS, and condom negotiation, and for a physical examination. The examination included both speculum and bimanual components. Specimens were collected for testing in the following order from the cervix: cervical Gram stain, Neisseria gonorrhoeae culture, $N$ gonorrhoeae by LCx, Chlamydia trachomatis by LCx, herpes simplex virus (HSV) by polymerase chain reaction (PCR), and human papillo- mavirus by PCR. The final four tests were obtained in one specimen collection tube supplied by Abbott Laboratories, Abbott Park, IL, USA. Vaginal specimens were collected with dacron swabs for the normal saline preparation, potassium hydroxide preparation, $\mathrm{pH}$ determination, whiff test, vaginal Gram stain, candida culture, and trichomonas culture (random order).

\section{LABORATORY METHODS}

Genital specimens were collected for immediate microscopic analysis and $\mathrm{pH}$ determination, in-house cultures for candida species (Sabouraud agar, obtained from Liofitchem diagnostici, Roseto Degli Abruzzi, Italy), $N$ gonorrhoeae (Thayer-Martin plates, prepared in-house from medium base and haemoglobin obtained from Bacto, Difco Laboratories, Detroit, MI, USA), and Trichomonas vaginalis cultures (InPouch TV, Biomed Diagnostics, San Jose, CA), ${ }^{15}$ and delayed testing for Chlamydia trachomatis (Abbott LCx probe system, Abbott Laboratories, Abbott Park, IL, USA), $N$ gonorrhoeae (Abbott LCx probe system, Abbott Laboratories, Abbott Park, IL, USA), and HSV (PCR, HSV-1 and HSV-2 primers obtained from Midland Certified Reagent Company, Midland, TX, USA) at the University of Michigan, and PCR testing for HPV infection at Wayne State University (Lucie Gregoire, PhD, Detroit, MI, USA). Quality assurance was maintained in the Bali laboratory by using trained laboratory technicians for all testing, testing known positives when available to assure the quality of the reagents, and comparing results of different methods of detecting the same organism (wet prep versus culture, $\mathrm{LCx}$ versus culture, etc) to further detect unexpected discrepancies. Quality control at the University of Michigan laboratories included routine inclusion of negative and positive controls. The diagnosis of bacterial vaginosis was estimated in two ways-using the Amsel criteria, and using Nugent's criteria on the Gram stain. The in-house cultures were inoculated on the media at the bedside, and were incubated at $35^{\circ} \mathrm{C}$, with a carbon dioxide candle jar used for $N$ gonorrhoeae and cultures. Candida cultures were read at 2 days, $N$ gonorrhoeae cultures at $1-3$ days, and $T$ vaginalis cultures at 2-10 days. $N$ gonorrhoeae cultures with Gram negative diplococci present on Gram stain of representative colonies were further evaluated with catalase and oxidase testspositive results on each of these was diagnostic of $N$ gonorrhoeae. Candida species and $T$ vaginalis were identified using 10-40× microscopy of representative colonies (candida) and samples of the culture media ( $T$ vaginalis). The specimens for molecular testing to be performed at the University of Michigan were inoculated into transport tubes supplied by Abbott Laboratories for use with the LCx test, were immediately frozen at $-4^{\circ} \mathrm{C}$, and were transported to a $-70^{\circ} \mathrm{C}$ freezer within 5 hours. Transportation to Michigan occurred quarterly with specimens remaining frozen on dry ice during transport. 


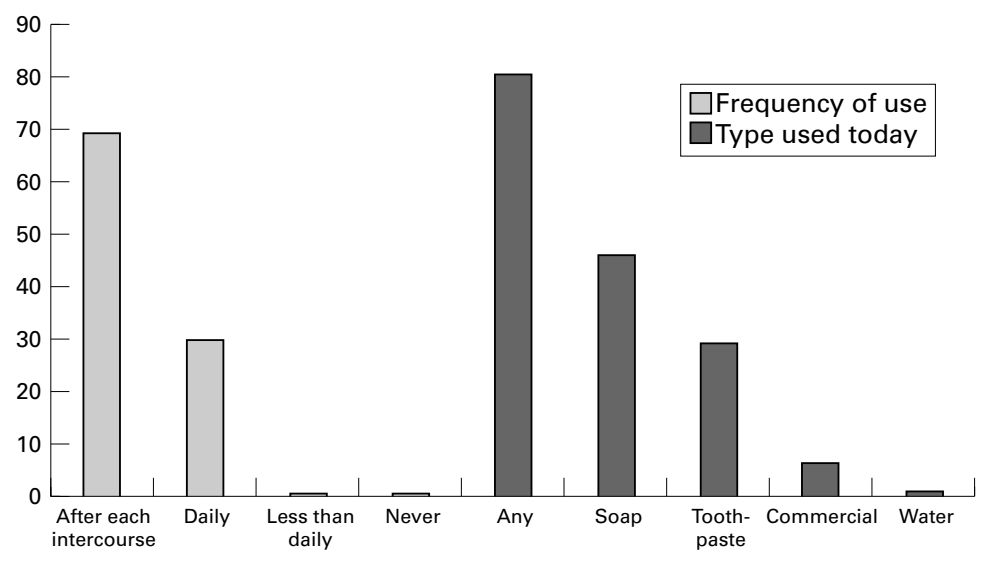

Figure 1 Frequency of use and type of genital cleanser used today.

Blood was drawn and tested in-house for syphilis using RPR, followed, if positive, by TPHA. Owing to reluctance of the women to have HIV testing performed and linked to their names, all specimens were unlabelled before sera were tested for HIV by the ELISA method and confirmed with western blot at the Naval Research Unit II in Jakarta, Java, Indonesia. However, HIV testing, with additional pretest and post-test counselling, was available to any woman who desired such testing-although none agreed to having these results linked to, or received by, them.

Syndromic diagnosis criteria, along with results of the immediate in-office microscopic results (high power microscopic examination of Gram stained vaginal and cervical specimens), were used for acute treatment decisions, followed by further treatment based on delayed results of laboratory tests. The questions regarding the vaginal cleanser use characteristics were added following round 1 when the presence of a very high vaginal $\mathrm{pH}$ in the majority of women during round 1 led to investigation of the use of administration of foreign substances in the vagina that might affect the $\mathrm{pH}$. Interviews with $10 \mathrm{CSWs}$ indicated use of vaginal cleansers was common, and hence detailed questions regarding this use were added at that time.

\section{DATA ANALYSIS}

Complete data on the interviews, the examinations, and on the majority of the infections were available using round 2 data alone. The viral data on the presence of HSV and of HPV at the cervix were determined at round 1 , but not at round 2 . Hence, only the women in round 2 who had also been present during round 1 , and had had tests for these infections were used to assess the associations between vaginal cleansing and these two infections.

The data were explored using frequency distributions for categorical variables and histograms and summary statistics for continuous variables. Univariate comparisons, using $t$ test and $\chi^{2}$ tests, were assessed for the association between the frequency of genital cleansing and the presence or absence of genital symptoms and signs, office laboratory data, and the prevalence of each genital infection. Odds ratios (OR) and 95\% confidence intervals (CI) were reported indicating the odds of each exposure in the women using cleansers after each intercourse compared with those using only daily or less. The associations between the women reporting they use genital cleansers to "avoid infection" with their knowledge and beliefs about STDs, AIDS, and condom use were similarly assessed, with odds ratios reflecting risk in women using cleansers to "avoid infection" compared with those who did not share this belief. Multivariate analysis was performed, using logistic regression, to assess the effect of potential confounders, including age, educational status, months as a sex worker in Bali, history of known STD, extent of reported condom use, and the number of clients in the past week.

\section{Results}

Data on 625 CSWs were collected between May and July 1998 for round 2 of the project. The women averaged 25.8 (SD 5.4) years of age, and had 4.9 (3.3) years of formal education. Most (93.9\%) were Muslims. Only $11.9 \%$ ( 25 women) had never been married, and $70.4 \%$ (440 women) were currently divorced. Parity averaged 1.5 (1.2). The women had worked as sex workers for an average of 13.5 (18.9) months, and had had an average of 3.7 (2.8) sexual clients the previous day. Of women who had worked in the past week, they reported the frequency of condom use in their sexual encounters during that time as $100 \%$ in 117 women $(22.2 \%)$, more than $50 \%$ but less than $100 \%$ in $321(60.8 \%)$, and less than $50 \%$ in 90 women $(16.0 \%)$.

Use of vaginal cleansers was very common among these sex workers, with very few $(<1 \%)$ reporting using cleansers less than daily (fig 1). The types of agents used varied, but over $90 \%$ had at one time used soap, toothpaste, or a combination of the two, with $70.1 \%(n=423)$ having used one or more of these on the day of the interview (fig 1). Many reported multiple reasons for using the cleanser, but $82.0 \%$ (509 women) indicated "to feel cleaner" was an important indication, with the second most common reason for use $(41.1 \%$, or 249 women) being "to prevent infection." Less than $5 \%$ reported using genital cleansers for treatment or prevention of odour, to make the vaginal drier, because others told them to, or because they perceived clients expected it. The frequency of vaginal cleansing was not associated with age, education, months having worked as a sex worker in Bali, or having had a history of STDs. However, women using cleansers after each client were less likely to report all their clients used condoms in the past week $(17.4 \%)$ compared with those who cleansed daily or less $(33.1 \%)(\mathrm{p}<0.0001)$.

The use of vaginal cleansers may have an effect on the symptoms experienced by the women, and on the findings present during the physical examinations. The associations between genital symptoms and using cleansers after each intercourse, compared with using them only daily or less, are given in table 1 . Women using genital cleansers after each intercourse were less likely than those using genital 
Table 1 Association between frequency of use of vaginal cleansers with symptoms reported

\begin{tabular}{lllll}
\hline Symptoms & $\begin{array}{l}\text { Use after each } \\
\text { intercourse } \\
\%(\mathrm{No})\end{array}$ & $\begin{array}{l}\text { Use daily } \\
\text { or less } \\
\%(\mathrm{No})\end{array}$ & p Value & OR $(95 \% \mathrm{CI})$ \\
\hline Vaginal discharge & $20.8(89)$ & $20.1(38)$ & 0.85 & $1.04(0.68,1.60)$ \\
Discoloured vaginal discharge & $4.9(21)$ & $15.3(29)$ & 0.00001 & $0.28(0.16,0.51)$ \\
Vaginal itching & $17.5(75)$ & $16.4(31)$ & 0.73 & $1.08(0.68,1.71)$ \\
Odour & $10.3(44)$ & $17.5(33)$ & 0.01 & $0.54(0.33,0.88)$ \\
Spotting & $3.5(15)$ & $7.9(15)$ & 0.02 & $0.42(0.20,0.88)$ \\
Genital sores & $14.3(61)$ & $21.7(41)$ & 0.02 & $0.60(0.39,0.93)$ \\
Tender inguinal nodes & $4.0(17)$ & $7.4(14)$ & 0.07 & $0.52(0.25,1.07)$ \\
Pain low abdomen & $32.7(140)$ & $27.5(52)$ & 0.20 & $1.28(0.88,1.87)$ \\
Painful urination & $14.5(62)$ & $14.8(28)$ & 0.92 & $0.97(0.60,1.58)$ \\
Discharge in a partner past month & $0.7(3)$ & $5.8(11)$ & 0.00008 & $0.11(0.03,0.42)$ \\
\hline
\end{tabular}

$\mathrm{N}=617$ for all variables, except one missing case for the variable regarding whether the partner had a discharge in the past month. was $23.2 \%$ (136/586). However, the validity of these criteria in this population is uncertain. In contrast, by normal saline preparation and Gram stain, $96.0 \%(576 / 600)$ of the vaginal specimens indicated a predominance of short rods and cocci, with only $21.2 \%$ (127/600) having long Gram positive rods present, suggesting the rate of $\mathrm{BV}$ may greatly exceed the $23.2 \%$ indicated by Amsel criteria. Of the 625 women studied, 238 women had been present in round 1 as well as round 2, and 198 had had HSV and HPV testing done in round 1 -of these $1 \%$ had active HSV-2 infection (two of 195 by PCR, none by examination), and $37.5 \%$ had HPV infection (74 of 198 by PCR, none by examination).

The association between the presence of each infection and whether the woman used a vaginal cleanser after each intercourse or only daily or less was determined (table 2). Only the presence of candida species on culture was associated with more frequent genital cleansing, with the presence of this organism increased in those cleansing after each client compared with those cleansing less often. The type of cleanser used (soap versus toothpaste) or using a genital cleanser on the day of examination was not associated with rates of any of the infections assessed (data not shown). Using logistic regression, the observed relations between the genital infections and the frequency of genital cleansing (table 2) were unchanged when the variables reflecting age, education, months working as a sex worker in Bali, history of STDs, and the number of clients yesterday were added to the model.

Women using genital cleansers to "avoid infection" may have different risk behaviours for acquiring STDs or HIV compared with those who do not believe that is a role for genital cleansers. In particular, they may differ in other risk factors for infection, including knowledge about STDs and AIDS, perceived vulnerability to infection, and frequency of use of condoms to prevent infection. The associations between these factors (recorded before the educational session) and reporting that they used vaginal cleansers to prevent infection are shown in table 3 . Among the women interviewed in round $2,571(92.4 \%)$ had heard of STDs, and $543(87.9 \%)$ had heard of AIDSboth prevalences were higher among women who believed that vaginal cleansing helped to prevent infection. Among those aware of STDs

Table 2 Association between the frequency of use of vaginal cleansers and the presence of genital infections *

\begin{tabular}{|c|c|c|c|c|}
\hline Infection (number with valid results) & $\begin{array}{l}\text { Cleansed after } \\
\text { each intercourse } \\
\%(\text { No) }\end{array}$ & $\begin{array}{l}\text { Cleansed } \\
\text { daily or less } \\
\% \text { (No) }\end{array}$ & $p$ Value & OR $(95 \% C I)$ \\
\hline HSV on cervix (195) & $0.7(1)$ & $1.8(1)$ & 0.52 & $0.41(0.3,6.65)$ \\
\hline HPV on cervix (198) & $38.3(54)$ & $35.1(20)$ & 0.67 & $1.15(0.60,2.18)$ \\
\hline Chlamydia trachomatis (598) & $38.9(157)$ & $40.5(70)$ & 0.72 & $0.94(0.65,1.35)$ \\
\hline Neisseria gonorrhoeae by LCx (596) & $57.5(231)$ & $55.8(96)$ & 0.71 & $1.07(0.75,1.53)$ \\
\hline Candida species on culture (601) & $28.9(118)$ & $17.8(32)$ & 0.005 & $1.87(1.21,2.90)$ \\
\hline Candida vulvovaginitis (Candida on culture plus symptoms) (598) & $11.0(45)$ & $9.4(17)$ & 0.58 & $1.18(0.66,2.13)$ \\
\hline Trichomonas vaginalis on culture (596) & $6.3(26)$ & $6.5(12)$ & 0.92 & $0.97(0.48,1.96)$ \\
\hline Bacterial vaginosis by Amsel criteria (578) & $22.4(90)$ & $23.7(42)$ & 0.83 & $0.93(0.61,1.41)$ \\
\hline Bacterial vaginosis by Nugent's criteria (592) & $95.9(395)$ & $96.1(173)$ & 0.89 & $0.89(0.39,2.31)$ \\
\hline Syphilis using RPR and TPHA (575) & $9.8(38)$ & $11.9(21)$ & 0.51 & $0.83(0.46,1.42)$ \\
\hline
\end{tabular}

^Data on HSV and HPV infection were available on the baseline specimens only. Only women who were tested in both rounds 1 and 2 were included in this analysis of these two infections. All other infections included data from round 2. 
Table 3 Association between knowledge and attitudes about STDs and AIDS and believing that using genital cleansers will help prevent STDs

\begin{tabular}{|c|c|c|c|c|}
\hline & $\begin{array}{l}\text { Used cleansers to prevent infection, } \\
\% \text { or difference in means (No) }\end{array}$ & $\begin{array}{l}\text { Did not use cleansers to prevent } \\
\text { infection, \% or difference in means (No) }\end{array}$ & $p$ Value & $\begin{array}{l}\text { OR or difference in } \\
\text { means }(95 \% \mathrm{CI})\end{array}$ \\
\hline \multicolumn{5}{|l|}{ STD factors } \\
\hline Heard of STDs & $96.0 \%(238)$ & $90.0 \%(333)$ & 0.01 & $2.64(1.29,5.42)$ \\
\hline Knowledge about STDs (scale 1 , low to 13 , high) & $6.60+2.31(231)$ & $7.43+2.41(321)$ & 0.000 & $0.83(-1.24,-0.43)$ \\
\hline Worried about getting STDS & $99.1 \%(229)$ & $99.7 \%(320)$ & 0.38 & $0.35(0.03,3.97)$ \\
\hline Believe STDs can be prevented by cleaning after sex & $57.6 \%(133)$ & $29.9 \%(96)$ & 0.000 & $3.18(2.23,4.53)$ \\
\hline Believe they are at risk for STDs & $2.2 \%(5)$ & $3.1 \%(10)$ & 0.50 & $0.69(0.23,2.04)$ \\
\hline Believe condoms can help prevent STDs & $91.6 \%(217)$ & $91.7 \%(299)$ & 0.95 & $0.98(0.54,1.79)$ \\
\hline \multicolumn{5}{|l|}{ AIDS factors } \\
\hline Heard of AIDS & $91.9 \%(228)$ & $85.1 \%(315)$ & 0.01 & $1.99(1.16,3.41)$ \\
\hline Knowledge about AIDS (scale of 1 , low to 23 , high) & $12.86+4.53(221)$ & $14.48+4.72(301)$ & 0.000 & $1.62(-2.43,-0.82)$ \\
\hline Worried about catching AIDS & $100.0 \%(228)$ & $99.0 \%(312)$ & 0.14 & $1.72(1.61,1.85)$ \\
\hline Believe AIDS is a fatal disease & $64.5 \%(147)$ & $72.4 \%(228)$ & 0.05 & $0.69(0.48,1.00)$ \\
\hline Believe medications can prolong life with AIDS & $33.3 \%(76)$ & $24.2 \%(76)$ & 0.02 & $1.57(1.07,2.28)$ \\
\hline Believe they are at risk for AIDS & $3.0 \%(6)$ & $4.8 \%(14)$ & 0.31 & $0.61(0.23,1.61)$ \\
\hline Believes condoms can help prevent AIDS & $90.3 \%(205)$ & $83.1 \%(260)$ & 0.02 & $1.90(1.12,3.23)$ \\
\hline
\end{tabular}

or AIDS, using genital cleansers in part to "prevent infection" was associated with the belief that STDs can be prevented by cleaning after sex, and that medications can prolong life with AIDS. However, women believing AIDS is a fatal disease were less likely to use genital cleansers to "prevent infection." Although most women stated they were "worried" about getting STDs and AIDS, very few felt personally at risk for getting these infections because the preventive measures they used (such as taking antibiotics regularly, taking "jamu" or traditional medicines, cleaning genitals, and using condoms). Women believing vaginal cleansing could prevent infection were more likely to state that condoms could prevent AIDS, but were no more likely to think STDs were prevented by condom use than were the other women. Reported condom use for "all" of their sexual encounters in the past week was uncommon $(22.4 \%)$ and did not differ in the two groups.

Multivariate analysis, using logistic regression, indicated that women using vaginal cleansers in part to "prevent infection," had a lower education level (number of years of formal education) than did women without this belief. Not associated with this belief were age, length of time having worked as a sex worker in Bali, history of STDs, frequency of condom use in the past week, or the number of clients seen the day before the interview. Including these variables in the model with each of the variables shown in table 3 resulted in the following differences: the lack of awareness of STDs, and the belief that medications could prolong the life of people with AIDS were no longer associated with the belief that vaginal cleansing would help prevent infection. The other associations were not altered by the multivariate model.

\section{Discussion}

The use of vaginal cleansers is a common practice worldwide. ${ }^{1-46}$ Yet, the practice is rarely evaluated in studies of women's genital infections and symptoms. The role that this cleansing may have on genital symptoms, clinical findings, laboratory testing results, or the incidence of infection has not been elucidated.

Vaginal cleansing may be associated with altered genital infection rates for disparate reasons. Insertion of foreign substances may result in alterations of the natural defences in the area (bacterial, immunological, $\mathrm{pH}$, and so on). Presence of a genital infection, with its concomitant vaginal symptoms, may foster the impression that cleansing is necessary and hence increase the likelihood of the behaviour. Conversely, genital cleansers might have an inhibitory effect on some genital pathogens and might decrease transmission rates. The physical activity of cleaning out the vagina also may play a part in removal of pathogens, or removal of semen that may be affecting transmission rates. Hence, whether vaginal cleansing is beneficial, detrimental, or a combination of the two, is unclear.

PREVALENCE OF GENITAL CLEANSING

Among the low priced commercial sex workers in the Denpasar, Bali area, most women were using soap, toothpaste, or a commercial douche preparation to clean the vaginatypically after each client. While the more prevalent reason for use was to feel cleaner, $39.7 \%$ also reported they performed this to decrease their risk of infection. Other populations around the world also use vaginal cleansers or douches routinely, such as sex workers and pregnant women in Africa, ${ }^{1} 16{ }^{17}$ American women in the United States, ${ }^{4}$ and abroad, ${ }^{2}$ and American adolescents. ${ }^{3}$ Hence, understanding factors associated with this practice and the effects of genital cleansing on genital infections may have implications for a large proportion of the adult world population.

\section{DOUCHING AND SYMPTOMS OR SIGNS}

The effect of douching on symptoms reported by women or on physical examination findings by medical personnel is unknown. Most women in our study douched at least daily, and hence comparison of symptoms and signs between those douching and those not was impossible. Despite the high rate of STDs in this population, only $20 \%$ reported vaginal discharge, although $31 \%$ reported low abdominal pain in the past month. Women douching after each intercourse compared with those douching less frequently did report less discoloured vaginal discharge, less vaginal odour, and less spotting, genital sores and painful inguinal lymph nodes. Hence, those douching frequently may be lacking the genital symptoms or signs that may in other populations indicate 
an increased probability of genital infections, leading to a decrease in diagnostic suspicion and accuracy.

GENITAL CLEANSING AND EFFECT ON VAGINAL BACTERIAL FLORA

Little is known about the effect of various types of vaginal cleansers on vaginal bacterial flora and on local mucosal immunity. ${ }^{18}$ Onderdonk et al found the use of a douche preparation containing acetic acid caused a transient reduction of the total bacterial counts, with most of the change attributable to the effect of washing the surface of the vaginal vault. In contrast, a povidone-iodine preparation caused a significant reduction in total bacterial counts compared with use of a physiological saline solution by the same subjects $(p=0.02)$. Following the reduction, the bacterial counts rebounded to even higher levels than baseline-more with the povidone-iodine than with the acetic acid douche. Little change in the qualitative makeup of the vaginal microflora occurred. The authors speculated that in some individuals, such changes in the vaginal flora might allow rapid proliferation of potential pathogens during the altered state, increasing the risk of associated infections.

Others have found an association between vaginal cleansing and the acquisition of bacterial vaginosis. ${ }^{719}$ Hawes et al found an increased risk of $\mathrm{BV}$ in women douching for cleanliness $(\mathrm{OR}=2.1,95 \%$ CI $1.0-4.3){ }^{7}$ A case-control study in London, UK demonstrated the strong association between the presence of bacterial vaginosis (BV), using Nugent's criteria, and genital cleansing practices (OR 3.5; 95\% CI 1.7-6.9). ${ }^{19}$ The implication this has on STD risk is strengthened by data regarding sex workers in Bombay, India, ${ }^{20}$ and in Mombasa, Kenya, ${ }^{17}$ in which lack of lactobacilli on culture or the presence of an abnormal background flora on Gram stain (present in $70 \%$ of women using genital cleansing methods ${ }^{17}$ ) was associated with an increased risk of HIV,${ }^{17}{ }^{20} \mathrm{~N}$ gonorrhoeae,${ }^{17}$ or $T$ vaginalis acquisition. ${ }^{17}$

In our study, it was uncommon to see a background vaginal flora with a moderate or heavy amount of lactobacilli type organisms present, and most women had a large number of short rods or curved rods present on Gram stain of the vaginal discharge. Hence, by Nugent's criteria, bacterial vaginosis was very common in this population, similar to the experience of others studying similar populations. ${ }^{17}$ Making the diagnosis of BV by Amsel's criteria did not appear to be accurate in this population because this population differed in several ways from the populations on whom the criteria were originally based. In our population, despite very common abnormal background flora, there were few positive whiff tests $(10.2 \%)$, clue cells were infrequent $(29.7 \%)$, and the vaginal $\mathrm{pH}$ was consistently high ( $>97 \%$ with $\mathrm{pH}$ of 4.8 or higher). Whether or not these characteristics are due to the chronic cleansing with high $\mathrm{pH}$ substances, such as soap and toothpaste, cannot be determined in this population.
GENITAL CLEANSING AND PREVALENCE OF STDs STDs were common among the low priced CSWs studied, with the $N$ gonorrhoeae prevalence $(57.3 \%)$ surpassing that of sex workers in several other countries. ${ }^{21-24}$ Conversely, their current prevalence of HIV seropositivity remained very low $(<1 \%)$, compared with the African populations. ${ }^{21}{ }^{23}$ Whether vaginal cleansers affect these rates is unclear. Evidence suggests some products applied to the vagina may be virucidal to potential genital pathogens. Spermicides containing nonoxynol-9 may inactivate enveloped viruses such as herpes simplex virus type 2 (HSV-2) and human immunodeficiency virus type 1 (HIV-1), but they are inactive against non-encapsulated viruses such as human papillomavirus (HPV). However, recent data suggest that an alkyl sulphate surfactant, sodium dodecyl sulphate (SDS), found in shampoos, detergents, and toothpaste, not only inactivates HSV-2 and HIV-1, but also inactivates HPV virus. ${ }^{12}$ The effect of these products on genital infections in vivo is unknown.

While in some populations, use of proprietary douching substances is the norm, in the women we studied, soap or shampoo were the typical genital cleansing agents used. In this population, we found no altered rate of infection with $C$ trachomatis, $N$ gonorrhoeae, HPV, syphilis, or $T$ vaginalis when women douched after each intercourse compared with those douching daily or less. Those douching more frequently were more likely to have candida species present in the vagina, although the rate of symptomatic candida vulvovaginitis was not different between the two groups. Whether the rates of infections would be altered if genital cleansing was not so prevalent in the vast majority of women studied is unknown.

Others have found the rates of STDs to be altered in women douching frequently. Joesoef et al found that the association with STDs was enhanced if the women douched before sex; adjusted odds ratio were 2.7 (95\% CI 1.0, 7.3) for douching with water and soap and 5.2 (95\% CI 1.6, 16.7) for douching with betel leaf or a commercial agent. ${ }^{5}$ Compared with women who never douched, women who always douched with betel leaf or a commercial agent had a substantially increased risk for having a sexually transmitted disease (adjusted odds ratio $9.4,95 \%$ confidence interval 1.8 to 50.3). La Ruche et al, studying pregnant women in Cote d'Ivoire, found women using vaginal cleansers to have fewer observed genital warts, but increased Ureaplasma urealyticum (associated with water douches), increased $C$ trachomatis (if douching with antiseptic solutions), and increased candida species infections. ${ }^{1}$ They similarly found an increased rich vaginal flora in women douching with soap, but less flora if douched with antiseptic. Gresenguet et al found women at an STD clinic in Central African Republic to be at increased risk for HIV infection and vaginal yeast when using a non-commercial douching product, but were at decreased risk for HIV when using a commercial product. ${ }^{6}$ 
Other genital problems that are associated with STDs have also been found to be increased in women who douche. These include the presence of PID $^{8-10}$ and ectopic pregnancies. $^{811}$ These studies comprised predominantly women in industrialised countries, where most vaginal cleansing involved the instillation of a fluid into the vagina, often under some force with a bag applicator, and these risks may or may not apply to genital cleansing performed with the soap or toothpaste on the finger - the technique performed by the CSWs we studied.

GENITAL CLEANSING AND KNOWLEDGE

REGARDING STDs AND AIDS

Previous data on this population of low priced sex workers indicate that awareness of STDs and AIDS has increased since the early $1990 \mathrm{~s}^{25}$ However, it is concerning that although the vast majority of women in the current study were "worried" about STDs and AIDS, very few considered themselves at risk for contracting these infections. Women using genital cleansers in order to "prevent infection" were more likely that those without this belief to have heard of STDS and AIDS, but were less knowledgeable about these infections. These same women had had less formal education than had women not using genital cleansing for this reason. Therefore, genital cleansing for infection prevention may be a marker of an insufficient knowledge base regarding these infections and personal risk of becoming infected.

\section{LIMITATIONS}

There are limitations to this study. Because the frequency of genital cleansing among the CSWs studied in Bali is very high, there was no "low frequency" group with which to compare. Because of this relative homogeneity, it is not possible to state whether the low frequencies of some infections (such as trichomonas vaginitis, HSV, HPV, and HIV infection) are related to the vaginal cleansing practices, or whether the frequencies of the most common infections (such as $N$ gonorrhoeae and $C$ trachomatis) are influenced by this practice. Nevertheless, differences between those cleansing with very high frequency (after each intercourse), and those of moderately high frequency (vaginal cleansing at least daily) do suggest some effects may be attributed to the cleansing practice. Further study in populations with greater variability in genital cleansing practices is needed.

This study was funded by NIMH grant no MH55942. We sincerely appreciate the assistance of Gung Adi for data entry and management for this project, and of Robin Williams for manuscript preparation

Contributors: All authors were involved in the study design, implementation of data collection, and manuscript preparation and review. In addition, BDR coordinated the documentation of
STDs, the data analysis, and the manuscript preparation; KF designed and modified the interviews to include genital hygiene documentation; and DNW coordinated the evaluation of the women in their places of work and at the clinics.

1 La Ruche G, Messou N, Ali-Napo L, et al. Vaginal douching: association with lower genital tract infections in African pregnant women. Sex Transm Dis 1999;26:191-6.

2 Stock RJ, Stock ME, Hutto JM. Vaginal douching. Current concepts and practices. Obstet Gynecol 1973;42:141-6.

3 Chacko MR, McGill L, Johnson TC, et al. Vaginal douching in teenagers attending a family planning clinic. $\mathcal{F}$ Adolescent Health Care 1989;10:217-9.

4 Aral SO, Mosher WD, Cates W Jr. Vaginal douching among women of reproductive age in the United States: 1988. Am women of reproductive age in the
$\mathcal{F}$ Public Health 1992;82:210-4.

5 Joesoef MR, Sumampouw H, Linnan M, et al. Douching and sexually transmitted diseases in pregnant women in Surabaya, Indonesia. Am F Obstet Gynecol 1996;174:115-9.

6 Gresenguet G, Kreiss JK, Chapko MK, et al. HIV infection and vaginal douching in central Africa. AIDS 1997;11: $101-6$.

7 Hawes SE, Hillier SL, Benedetti J, et al. Hydrogen peroxideproducing lactobacilli and acquisition of vaginal infections. F Infect Dis 1996;174:1058-63.

8 Zhang J, Thomas AG, Leybovich E. Vaginal douching and adverse health effects: a meta-analysis. Am f Public Health 1997;87:1207-11.

9 Foxman B, Aral SO, Holmes KK. Interrelationships among douching practices, risky sexual practices, and history of self-reported sexually transmitted diseases in an urban population. Sex Transm Dis 1998;25:90-9.

10 Jossens MO, Eskenazi B, Schachter J, et al. Risk factors for pelvic inflammatory disease. A case control study. Sex Transm Dis 1996;23:239-47.

11 Kendrick JS, Atrash HK, Strauss LT, et al. Vaginal douching and the risk of ectopic pregnancy among black women. Am $\mathcal{F}$ Obstet Gynecol 1997;176:991-7.

12 Howett MK, Neely EB, Christensen ND, et al. A broad-spectrum microbicide with virucidal activity against sexually transmitted viruses. Antimicrob Agents Chemother 1999;43:314-21.

13 Maguire RA, Zacharopoulos VR, Phillips DM. Carrageenan-based nonoxynol-9 spermicides for prevention of sexually transmitted infections. Sex Transm Dis 1998;25:494-500.

14 Patton DL, Kidder GG, Sweeney YC, et al. Effects of nonoxynol-9 on vaginal microflora and chlamydial infection in a monkey model. Sex Transm Dis 1996;23:461-4.

15 Draper D, Parker R, Patterson E, et al. Detection of Trichomonas vaginalis in pregnant women with the nPouch TV culture system. F Clin Microbiol 1993;31: $1016-8$.

16 Morar NS, Ramjee G, Abdool Karim SS. Vaginal insertion and douching practices among sex workers at truck stops in KwaZulu-Natal [letter] [published erratum appears in $S$ Afr Med F 1998;88:733]. S Afr Med F 1998;88:470.

17 Martin HL, Richardson BA, Nyange PM, et al. Vaginal lactobacilli, microbial flora, and risk of human immunodeficiency virus type 1 and sexually transmitted disease acquisition. F Infect Dis 1999;180:1863-8.

18 Onderdonk AB, Delaney ML, Hinkson PL, et al. Quantitative and qualitative effects of douche preparations on vaginal microflora [see comments]. Obstet Gynecol 1992;80: 333-8.

19 Rajamanoharan S, Low N, Jones SB, et al. Bacterial vaginosis, ethnicity, and the use of genital cleaning agents: a case sis, ethnicity, and the use of genital cleaning agen

20 Bhave G, Lindan CP, Hudes ES, et al. Impact of an intervention on HIV, sexually transmitted diseases, and condom use among sex workers in Bombay, India. AIDS 1995;9:S21-30.

21 Steen R, Vuylsteke B, DeCoito T, et al. Evidence of declining STD prevalence in a South African mining community following a core-group intervention [see comments]. Sex Transm Dis 2000;27:1-8.

22 Mulanga-Kabeya C, Morel E, Patrel D, et al. Prevalence and risk assessment for sexually transmitted infections in pregnant women and female sex workers in Mali: is syndromic approach suitable for screening? Sex Transm Inf 1999;75: approa.

23 Nzila N, Laga M, Thiam MA, et al. HIV and other sexually transmitted diseases among female prostitutes in Kinshasa. AIDS 1991;5:715-21.

24 Laga M, Alary M, Nzila N, et al. Condom promotion, sexually transmitted diseases treatment, and declining incidence of HIV-1 infection in female Zairian sex workers. Lancet 1994;344:246-8.

25 Ford K, Wirawan DN, Fajans P. AIDS knowledge, risk behaviors, and condom use among four groups of female ex workers in Bali, Indonesia. f AIDS 1995;10:569-76. 